\title{
Reflexiones en torno a lo indecible y lo inenarrable. Una propuesta metodológica para el estudio de experiencias traumáticas.
}

\author{
Reflections on the unspeakable and the inexpressible. A proposed methodology for the study of traumatic \\ experiences \\ Rosaura Barrios \\ Instituto de Investigaciones Sociales y Humanasn, Universidad Nacional de Mendoza- (IISH/UNaM), Argentina \\ rocha_barrios@yahoo.com.ar
}

\section{ReSUMEN:}

En el estudio de narrativas que tienen que ver con la experiencia dolorosa (E. D.) de la violencia sexual y/o las relaciones que construimos entre/con las personas que intervienen en una investigación tienen toda una dimensión sensitiva- corporal imposible de eludir a la hora de construir los materiales de análisis. En este artículo me propongo, por un lado, reflexionar- justamente- sobre estas relaciones, que se construyen en campo, incorporando al corpus de análisis los registros experienciales del/a investigador/a a la hora de reflexionar para, por otro lado, poner al alcance del lector una propuesta metodológica para el abordaje de narrativas que tienen que ver con experiencias dolorosas. A partir de (y gracias a) esta vigilancia epistemológica, buscamos poner en crisis algunas técnicas de recolección de datos tradicionales y preguntarnos por otras maneras de abordar-pensar-sentir y sistematizar relatos de experiencias dolorosas.

Palabras Clave: Experiencias Dolorosas, Trabajo de Campo, Metodología.

\section{Abstract:}

In the study of narratives that deal with the painful experience (ED ) of sexual violence, the relationships built between / with persons involved in an investigation are all an impossible body sensitive- elude dimension in building materials analysis. In this article I propose, first, think about these relationships that are built in the field work, incorporating experiential corpus analysis records / a researcher / at time to reflect, on the other hand, make available reader a methodology for addressing narratives that deal with painful experiences. From (and thanks to) this epistemological vigilance we want to put in crisis some traditional techniques of data collection and questions about other ways of thinking-feeling - address and systematize accounts of traumatic experiences.

KeYwords: Painful Experience, Field Work, Methodology.

\section{INTRODUCCIÓN}

A partir del estudio de relatos para mi tesis doctoral (FPyCS- UNLP), que tienen que ver con la Experiencia Dolorosa (ED) que origina la violencia sexual narrada en distintas situaciones comunicativas, es que nos vimos en la necesidad de revisar las técnicas de recolección de datos disponibles. Fue ante la imposibilidad de registrar situaciones o experiencias y en el reconocimiento de fisuras en el entramado analítico que buscamos otros tipos de registro y acompañamiento durante la etapa del trabajo de campo.

Para el presente trabajo hicimos un recorte del análisis y del campo, y presentamos algunas reflexiones en torno a las condiciones de enunciación de relatos que tienen que ver con el abuso sexual (durante la infancia) en una ONG de la provincia de Buenos Aires, que no sólo asiste y trata estas problemáticas, sino que las estudia también. Estas líneas constan de tres partes. En primer lugar el mapa reflexivo que atravesamos (y que nos atraviesa) en el abordaje de estas narrativas de experiencias traumáticas: ¿qué relación tiene la experiencia de vida del/a investigador/a con su tema de investigación?; ¿̇tienen "utilidad” epistemológica las emociones que se desprenden de la práctica de campo?; ¿cuál es nuestro compromiso con el/la otro/a?; ¿ puede 
una posicionarse en campo, romper con esa supuesta "objetividad" en un plano etnográfico y epistémico?; ¿cómo convertir un problema social en un problema teórico- epistemológico?. Estas preguntas son las que nos guiarán a la segunda parte de este trabajo que consta de una propuesta metodológica concreta, pensada desde la comunicación, para el abordaje de relatos de experiencias dolorosas que tienen que ver con la violencia sexual en la infancia. Esta propuesta pone el ojo no sólo en las herramientas y situaciones de recolección de datos, sino en las mismas condiciones de reflexión de estas narrativas científicas. ¿Qué disciplinas pueden ayudarnos en el armado de una metodología acorde a las preguntas que desprendemos del objeto? Finalmente, la tercera parte consta del estado de las reflexiones hasta hoy: sabemos que siempre se mueven, cambian, mutan y migran, y quisimos que esos movimientos estuvieran presentes en forma de preguntas para seguir pensando/amasando este entramado analítico/metodológico.

\section{ACERCA DE LA INVESTIGACIÓN}

Esta investigación es parte de una tesis de doctorado (Comunicación- FPyCS/ UNLP) financiada por CONICET (en ese momento con sede de trabajo en el IIGG/UBA). El recorte del campo que dio vida a estas reflexiones datan del 2012-2014 en una ONG de la provincia de Buenos Aires que asiste, trata y posee un grupo de estudios sobre la problemática del abuso sexual en la infancia. El principal objetivo fue reflexionar acerca de las condiciones de producción de narrativas experienciales que tienen que ver con la violencia sexual sufrida durante la infancia en distintas situaciones comunicativas. Si bien lo aquí presentado es un recorte del abordaje metodológico de la tesis, estas reflexiones buscaron ser exhaustivas, se iniciaron en las mismas condiciones de producción de estos análisis, pasaron por la construcción de una metodología para este objeto específico y finalizan en la reconstrucción del entramado analítico. Es decir, en el mapa de preguntas que guiarán el análisis.

Para este trabajo en particular (y a raíz de las reflexiones de la tesis doctoral) buscamos presentar una metodología de abordaje que dialogue con las formas de acceso que tiene esta organización en particular a los relatos de experiencias dolorosas de las personas que asisten a la ONG en calidad de víctimas de violencia sexual. En todo momento buscamos articular el abordaje de esta organización con las técnicas de recolección de datos disponibles. La situación comunicativa que nos permitió acceder a los relatos es el informe en formato forense que se desprende de la Evaluación Psicodiagnóstica (EP). El mismo busca reconstruir la psiquis de ese niño o niña hasta ese momento (si ese niño/a sufrió algún tipo de abuso la evaluación y sus indicadores dirá). Cada evaluación consta de ocho encuentros en cámara gesell con las profesionales que de manera posterior elaboran este informe para ser elevado a la autoridad competente. Todos los casos utilizados para esta investigación son casos judicializados que datan de hace dos o tres años -al momento de realizar el trabajo de campo (esto es a modo de preservar aún más las identidades de las personas que intervienen en la investigación)-, y cuentan con las autorizaciones de los/as adultos/as responsables, como así también de las autoridades de la ONG (esta última autoriza sólo a los fines investigativos). Cabe mencionar que los avances que fueron surgiendo de estos registros y reflexiones se pusieron al servicio de la mesa de estudios interdisciplinarios de Abuso Sexual con la que cuenta la organización.

Los análisis que se desprenden de esta metodología corresponden a la lectura y trabajo con los informes forenses, la participación en la mesa de estudios interdisciplinares, y encuentros con el equipo de salud que asistió a los niños y niñas. 


\section{Tres Movimientos para empezar a Pensar una metodología para ED}

\section{UNO. El cuerpo al relato}

Al tomar contacto con la problemática del abuso sexual, el trabajo requirió (como todos los temas de investigación) una preparación bibliográfica exhaustiva. Pero al momento de ingresar al campo, el cuerpo se hizo presente con todos sus sentidos y sentires. Yo suponía que la preparación que nos faltaba era teórica, pero había algo en el plano de lo sensitivo que no estuvimos preparadas para afrontar. Si bien estas impresiones superan la propuesta de este artículo, compartimos algunos interrogantes de campo para iniciar este recorrido metodológico:

¿Podemos desde las ciencias sociales y en nombre de la labor científica escuchar y enfrentar relatos sobre lo más doloroso y vergonzoso, como lo son los relatos de abuso sexual intrafamiliar? ¿A nombre de quién y a cambio de qué nos enfrentamos a ellos? ¿Cuál es la manera menos invasiva/violenta y revictimizante para acceder a estos relatos experienciales?

Cuando la pregunta por la "ética" profesional empezó a aflorar, al inicio del trabajo de campo, y las técnicas de recolección de datos tradicionales me empezaron a fallar, no tuve dudas de que debía abordar este problema desde otro lugar. Hasta ese momento pensaba que la preparación que me faltaba como investigadora era más bien teórica, metodológica quizás... estaba cerca de detectar otra urgencia: el cuerpo (de la investigadora) no estaba preparado para ponerse frente a tanto dolor ajeno, a tanta vergüenza, intimidad y repugnancia. Había algo en el plano de lo experiencial que faltaba.

Fue en esta instancia que el problema y la urgencia de reflexionar sobre la sensación emocional y corporal que estaba sintiendo en ese momento al escuchar (y que volvía con más frecuencia a medida que pasaban los días), no la podía evadir. Una reflexividad narcisista, dirá Rosana Guber, una reflexividad que habilita a la pregunta por la figura del investigador y sus emociones en campo: ¿son acaso materiales sensibles de ser incorporados al corpus de análisis? Como dirá Figari (2010), esa relación amorosa con el otro capaz de ser consciente del mapa de relaciones y de reconstruir ese tejido en el que estamos inmersas también como investigadoras. Con sus peligrosos límites y concesiones, esta perspectiva vino a ordenar bastante mis notas y diario de campo. Fue el único pasillo que encontré con la suficiente luz para acomodar tanta idea apiñada.

Como decíamos, Carlos Figari hace su propuesta metodológica con base en el concepto de conocimiento situado: describir, analizar, interpretar a partir de la relación entre el investigador y el otro como sujeto-sujeto, en lugar de la "falaz" relación sujeto-objeto, planteo propio de la investigación en las ciencias. La propuesta de Figari para el trabajo intelectual es una instancia que supera la supuesta objetividad que se pretende desde las ciencias sociales, la relación cuerpo-cuerpo - va a decir- supone la construcción de una relación afectiva con el otro, de comprensión mutua. Esto significó para mi trabajo tener muy presentes mis miedos, mis desventajas, y por supuesto mis prejuicios. Esa premisa que reza que todo investigador debe estar desprovisto de prejuicios a la hora de entrar a campo la cambiaríamos: tuvimos muy presentes todas las contradicciones propias de una mujer joven que recién empieza a indagar. La ruptura fue más fuerte, el extrañamiento, la novedad. Y es a esto que refiere Figari: pensarnos en función al otro, ese otro que también es una en ese momento.

Por esta construcción "afectiva" con el otro que menciona Figari nos dimos cuenta de que las tradicionales "técnicas de recolección de datos” no me alcanzaron para registrar y reconstruir la trama de aquellos días.

Hablar de "observación participante" sería dar cuenta de nuestra disposición general, pero no de las actividades concretas que llevamos a cabo en el campo; hablar de "entrevista en profundidad" supondría evocar una situación que pocas veces se nos planteó con tanta nitidez y delimitación; y referirnos a información y a informantes nos retrotraería a una objetivación con las que nos vinculamos que no estaríamos dispuestas a admitir porque no da cuenta de qué sentimos y cómo vivimos nuestros trabajo de campo con otras personas (Guber, 2015, p. 15). 
Tal como lo plantea Guber, necesitaba registrar, plasmar, codificar los distintos términos en que entablamos relaciones con nuestros interlocutores, recuperar de alguna manera esos días en que todo era nuevo, disruptivo, movedizo. Necesité herramientas, técnicas y habilidades que me permitiesen recuperar las lógicas que empezaba a conocer a partir del trabajo de campo etnográfico, entendido como relación social y como articulador de situaciones de interacción (Guber, 2014).

Ya en una segunda parte del trabajo de campo -que no la incluimos aquí pero que quisimos que sus interrogantes estuvieran presentes para pensar esta relación cuerpo de la investigadora/cuerpos investigadosse buscó correr esos límites metodológicos, ir un poco más allá para preguntarnos hasta dónde el cuerpo del investigador se presta/soporta/aguanta estas tristezas ajenas; ¿es posible la producción de conocimiento científico en estos "peligrosos" límites emocionales y físicos de los sujetos que intervienen en una investigación y, por qué no, del propio investigador?; ¿qué rol juega el secreto en estas tramas científicassociales de interacción?; ¿es posible investigar lo "no- dicho"? El secreto, justamente, viene a "resolver" comunicacionalmente el horror de contar y volver a vivir las características de los delitos sexuales que tienen algo (o todo) de indecible, inexplicable (para quien escucha y para quien cuenta), tiene todo de terror, es algo que- aparentemente- no puede ser dicho. Con toda la atención puesta allí, el cuerpo se prepara para escuchar algo terrible, siempre en la eterna encrucijada ética y profesional de abordar con mucho respeto y sensibilidad la tarea no sólo de escuchar y leer estos relatos, ponerles rostro, sino también de darles un tratamiento riguroso, científico y responsable. Hacía allí vamos.

\section{DOS. Las condiciones de reflexión de las preguntas de investigación}

Preguntarnos por las condiciones de enunciación o por la situación comunicativa que atraviesa/parte/ delimita las preguntas de investigación -que darán forma al problema de investigación- es preguntarse, también, por el contexto del investigador. Para pensar esto, Haber y Figari retoman los apuntes de Haraway, quien cree firmemente en localizar la posición desde donde se enuncian los análisis; si bien la identidad autoidentidad- (1999) no produce ciencia, el posicionamiento crítico sí. La objetividad es transparentar quién habla, desde dónde, bajo qué perspectiva, con qué influencias. Así los estudios de género vienen a sostener la trama metodológica de este trabajo, con los aportes de Haraway y su concepto de conocimiento situado.

Boaventura De Sousa Santos (2009), Irene Vasilachis de Gialdino (2006) y Alberto Leonardo Bialakowsky (2009) son autores que sin hablar de conocimiento situado, también trabajan con varios de sus postulados, en especial, con la crítica a la mirada hegemónica de las ciencias. Ellos señalan la importancia de contextualizar el conocimiento, de pensarlo como un proceso que implica prácticas, no sólo metodológicas sino también éticopolíticas, de reconocer y considerar como iguales a los sujetos conocidos, a los sujetos de estudio. Seguimos con De Sousa Santos, que desarrolla cómo en el acompañamiento de nuestros problemas de investigación nos encontramos muchas veces con teorías y conceptos que no son los más indicados y eficaces para permitir abordarlos en profundidad. Así “[cada vez] resulta más claro que las teorías, los conceptos, las categorías que usamos en las ciencias sociales fueron elaborados y desarrollados entre mediados del siglo XIX y mediados del siglo XX en cuatro o cinco países: Francia, Alemania, Inglaterra, Estados Unidos e Italia” (De Sousa Santos, 2009, p. 137). Es así como importamos teorías y categorías sociales legitimadas en el campo científico (de otros países, de otras regiones, de otras realidades), incongruentes con nuestro contexto. Otro de los problemas que destaca es "que las ciencias sociales son monoculturales, es decir que por detrás de los conceptos está la cultura occidental, y resulta problemático aplicar estos conceptos a realidades no occidentales" (2009, p.138). De allí la relevancia de los estudios poscoloniales en la actualidad.

Esas tensiones y obstáculos son afrontados con creatividad, "lo cual implica un trabajo de reflexividad (Guber, 2004), de diálogo y exposición” (Bialakowsky, 2009, p. 187) entre los investigadores y los sujetos de estudio "en el proceso de constitución en coproductores de conocimiento y de conformación de una voluntad 
colectiva por conocer y comprender" (Bialakowsky, 2009, p. 187). Este trabajo de reflexividad quizás sea la puerta que lleve a incorporar los registros experienciales de cada investigador/a. Recuperar, justamente, estas narrativas personalísimas es la propuesta del trabajo, ¿cómo esa vigilancia epistemológica contribuye a localizar no sólo el problema de investigación sino también los contextos de enunciación de las preguntas de investigación?

En la misma línea, Irene Vasilachis de Gialdino (2006) postula una epistemología del sujeto conocido como "fundamento de la investigación cualitativa y encaminada a reconocer la comprensión cooperativa del conocimiento en ciencias sociales" (2006, p. 1). "Esta propuesta se centra en la práctica real, situada, basándose en un proceso interactivo en el que intervienen el investigador y los participantes” (2006, p. 4), donde ambos interactúan, son transformados y se ven afectados; más aún cuando se indagan y rememoran experiencias ligadas al temor, la angustia, la violencia y el desamparo. El investigador como persona situada en un determinado contexto "debe tener presente que sus valores, perspectivas, creencias, deseos, expectativas influyen en la percepción y en la construcción de la realidad que estudia, y que la experiencia vivida es también una experiencia corporeizada" (Vasilachis de Gialdino, 2006, p. 8), y también que él mismo es una fuente de datos. Estas indicaciones forman parte de lo que la autora llama la epistemología del sujeto conocido, para diferenciarse de la epistemología del sujeto cognoscente. En el proceso de conocimiento que propone esta última, "el sujeto cognoscente queda como actor primordial, manteniendo la distancia necesaria como para asegurar la objetividad de su conocimiento, y suponiendo una diferencia que lo separa del sujeto conocido y que lo preserva de ser, él también, transformado durante dicho proceso”. En cambio, la epistemología del sujeto conocido

viene a hablar allí donde la Epistemología del sujeto cognoscente calla, muta o limita, e intenta que la voz del sujeto conocido no desaparezca detrás de la del sujeto cognoscente, o sea tergiversada como consecuencia de la necesidad de traducirla de acuerdo con los códigos de las formas de conocer socialmente legitimadas (Vasilachis de Gialdino, 2006, p. 17).

En este sentido, el sujeto conocido es una "parte activa en la construcción cooperativa del conocimiento y una presencia no oscurecida o negada, sino integralmente respetada en la transmisión de este" (Vasilachis de Gialdino, 2006, p. 18).

En este entramado de propuestas recuperamos la centralidad de la interpretación en la investigación cualitativa, la (re)flexibilidad de sus métodos y análisis tanto como la sensibilidad que se desprende del contexto en que los datos son "coproducidos" (Bialakowsky, 2009), siempre en forma "conjunta" con un otro que es parte activa en la construcción de conocimiento (Vasilachis De Gialdino, 2006).

Este registro corporal/ sensitivo/ escrito/ hablado del contexto de reflexión de las preguntas de investigación es el punto de partida para empezar a ordenar las notas de campo. La reflexividad es gran llave para desenredar estas tramas sin subestimar los relatos de experiencias traumáticas. Es, quizás, la manera de traducir experiencia sensitiva en investigación en materiales sensibles de ser analizados, ¿es posible investigar la experiencia dolorosa del abuso sexual?

\section{TRES. Una metodología para un campo fuertemente intervenido}

¿Cómo trabajar con los materiales de análisis teniendo en cuenta sus características?; ¿qué tratamiento merecen los relatos de experiencia traumática?; ¿cómo pensar un campo fuertemente intervenido por discursos de corte legal, psicológico, del campo de la criminalistica y el trabajo social?; ¿qué herramientas tomar de cada disciplina para construir el mapeo del trabajo de campo?; ¿cómo se escriben las notas de campo teniendo en cuenta todos los cuidados que merece el trato con población vulnerada?; ¿qué aporte podemos hacer desde la comunicación a estas tramas?

Este apartado problematiza cuestiones éticas y metodológicas que nos explotaron en el campo, dado es que las decisiones metodológicas tomadas en relación a experiencias que denigran y atentan contra la 
integridad humana deben ser cruzadas fuertemente con una ética investigativa rigurosa. Debemos entender la ética de la investigación "como la responsabilidad que debe tener la ciencia y más concretamente los científicos, hacia los sujetos de investigación y la sociedad en general” (Achío Tacsan, 2003, p. 85). Si bien parte de principios básicos que todo/a investigador/a debiera tener al iniciar una investigación que involucre a personas, hablamos mas bien de un compromiso social de cuidado, respeto y equilibrio (en términos de ejercicio de poder) hacia las personas con las que trabajamos. Son planteos teóricos y metodológicos en torno a la escucha, la observación y el silencio, que componen un mapeo posible para el estudio de ED que tienen que ver con el abuso sexual y la violación. Es -también- una puesta en tensión de la posición política de poder que ocupa el investigador en estas tramas, el uso de algunas herramientas de recolección de datos, y la necesidad de entablar diálogos y puentes con otras disciplinas con trayectoria en estos estudios. Con estos principios como norte y práctica encaramos y cuestionamos el trabajo de campo.

¿Cómo lograr una investigación que cumpla con los requisitos y criterios de rigurosidad científica sin intervenir de manera abrupta en la organización? Esto es tratar de empalmar los tiempos de las organizaciones con los del trabajo de campo. Sin apurar o trasladar a nuestro contexto esos relatos, el trabajo exploratorio fue fundamental para identificar estas dinámicas. ¿Por qué no abordar el problema desde las particularidades de la ONG con sus formatos del contar y narrar y sus géneros? ¿Cómo era contada la experiencia del abuso en esa ONG, con esas reglas, con esos tiempos y formatos?

El abordaje de ED expone no sólo a los/as entrevistadas a un estado de vulnerabilidad altísima, sino que también expone al investigador a una infinidad de encrucijadas éticas, políticas y filosóficas, que deberá sortear (o no) para llegar (o aproximarnos) a los objetivos planteados. La preocupación por encarar la escucha atenta y respetuosa es un interrogante no menor a la hora de encarar el trabajo, y justamente por la seriedad con la que se pretende encarar la labor científica es que resulta necesario dialogar con disciplinas como la psicología, la sociología y el trabajo social. Disciplinas que cuentan con herramientas, pero, sobre todo, con debates para el manejo de testimonios de experiencias dolorosas, las emociones personales y la relación entre víctimas y profesionales intervinientes en la escucha. La consulta con otros profesionales para trabajar el anonimato y la confidencialidad de una manera más rigurosa se vuelve fundamental en sintonía con organismos nacionales o internacionales que ya cuentan con un conjunto de procedimientos ante estos casos.

\section{¿Una metodología para el dolor?}

Por lo dicho en el apartado anterior es que no utilizamos como herramienta de recolección de datos la entrevista en profundidad, convencidas de que no contamos con insumos personales para afrontar el caudal de relatos de una víctima "sobreviviente" de abuso sexual, ni la formación para contener en caso de que el recuerdo desate sentimientos de tristeza o angustia.

...el trabajo de campo no consiste en la aplicación de métodos claramente definidos desde la academia con sus aulas y oficinas. Consiste en un reconocimiento más amplio de los términos en que entablamos relaciones con nuestros interlocutores y que nos permiten conocerlos recuperando sus perspectivas acerca de las cuestiones que les preocupan, les interesan, los motivan, los enorgullecen y los avergüenza (Guber, 2014, p. 15).

En línea con el planteo de Rosana Guber, el trabajo de campo y su intervención fueron pensados específicamente para este objeto de estudio. Buscamos las herramientas más adecuadas para empezar a pensar el problema sin ser intrusivas, apuradas o violentas en términos de autoridad etnográfica y de esta supuesta impunidad que tenemos desde las ciencias para realizar cualquier tipo de preguntas que competan a nuestro trabajo.

Este trabajo de campo de fuerte corte etnográfico -entendido como una perspectiva de conocimiento que aspira a comprender los fenómenos sociales desde el punto de vista de sus protagonistas- es pensado como relación social y como articulación de las situaciones de interacción (Guber, 2014). Como escenario "donde 
el investigador pone en interlocución sus categorías teóricas y prácticas de académico y de ciudadano con las categorías y prácticas nativas" (Guber, 2013, p. 59) para pensar, al mismo nos servimos de otras disciplinas para confeccionar su protocolo de acceso e intervención. Por un lado, de la psicología tomamos el manejo del anonimato y la confidencialidad, que si bien implica reflexiones que involucran a cualquier disciplina social, realizan un manejo distinto de las mismas, que no podría calificarse en términos de "mejor", pero sí de más adecuada, conocida y propia de este contexto. De la criminalística y el derecho tomamos el tratamiento y comprensión de documentos que refieren a causas legales en curso cuando hay niños, niñas y adolescentes involucrados, dado que siempre hay una denuncia de por medio. Tuvimos que aprender ese registro y su manejo en estos casos. De la antropología tomamos la categoría de reflexividad, que ordenó las notas de campo y su posterior escritura:

El concepto de reflexividad que se evoca en la etnometodología desarrollada por Harold Garfinkel (1967) e inspirado en la fenomenología propuesta por Alfred Schütz fue central para pensar el trabajo de campo y la etnografía. Se trata del papel constitutivo que ejercemos en cualquier ambiente al actuar y enunciar. (...) En este intersticio, que se crea y recrea en la relación, trabaja el antropólogo. No sólo reconociendo los procedimientos reflexivos de los nativos sino también los procedimientos propios, porque es en esa relación donde se produce sentido y se hace posible la comprensión en términos antropológicos, una comprensión susceptible de ser relatada que involucra siempre formas variables de invención y creatividad. Considerando el papel constitutivo de la reflexividad es posible reconocer las limitaciones que resultan al defender la idea de que existe una "realidad" separada del "sujeto" cognoscente, y de que ambos interferirán mutuamente (Renoldi, 2014, p. 131).

La literatura, el cine y la fotografía también contribuyeron a la comprensión de los relatos. No había palabras para contar, ni contábamos con imágenes para representar(nos) esos relatos. Tuvimos que aprender a trabajar con los silencios y el secreto. Este último, como categoría y práctica, fue un nudo troncal en el trabajo que obligó a virar el timón en su momento hacia otras estrategias metodológicas que lo contemplen en su complejidad. En cuanto a esto, "más que preguntarnos si lo que se nos ha dicho es cierto o no lo es, conviene averiguar qué significa y cuáles son las implicancias posibles de lo que se dice (e inferir lo que se calla)" (Guber, 2001, p. 243).

Aun pudiendo pensar en todo lo que significa para el etnógrafo entender el secreto en su dimensión sociológica, no deja de ser un conflicto para su trabajo saber cómo proceder con informaciones que le son contadas de forma confidencial por quienes saben que a él le interesa conocer y comprender sus vidas y sus cotidianos (Renoldi, 2014, p. 134).

En este sentido, se produce un doble movimiento: el investigador elige la información y recorta los datos y, a su vez, el campo elige qué mostrarle al investigador al punto que éste no sabe qué es o qué no es un dato a priori (Guber, 2013). La vigilancia sobre qué registrar y qué no registrar estuvo presente durante todo el trabajo. Fueron estas disciplinas las que nos ayudaron a descifrar qué y cómo registrar en el diario de campo sus experiencias en relación al abuso. Con respecto a este punto y a la incorporación de la reflexividad para pensar todo el proceso de investigación

...se trata de partir de la base de que no describimos 'realidades', sino que apenas podemos describir aquello que constituimos de manera activa en una red de relaciones heteromorfa y heterogénea que no tiene límites dados en sí, y que se constituye en la experiencia (Renoldi, 2014, p. 131).

\section{La escuch a y la observación}

Con la particularidad de este campo ajustado, limitado, y que lidia con un discurso institucional hipercodificado como el de las ONGs, cabe preguntarnos: ¿cómo movernos dentro de estas simplificaciones, limitaciones?; ¿cómo articular los objetivos personales de investigación con los comunes que nuclean a las personas que trabajan en una ONG?; ¿cómo empalmar los intereses científicos con los de las personas que 
forman parte de una investigación?; ¿ ¿cuál es la metodología y herramientas más adecuadas para el tratamiento de estos datos?

Cuando el callejón se hizo más estrecho y las técnicas de recolección de datos tradicionales no me bastaban para registrar todo lo que ese campo estaba provocando(me), devolviendo(me), mostrando(me), se hizo necesario incorporar otras lecturas, otras formas de abordar esos relatos. Todos los trabajos que abordan la experiencia dolorosa (y traumática en términos psicológicos) a través de testimonios escuchados y registrados en contextos institucionales por fuera de una investigación científica, y que ponen la mirada en conflictos bélicos (Colombia, Perú) y en las últimas dictaduras cívico- militares- eclesiásticas (América Latina en general con el Plan Cóndor) fueron mis referencias en esta nueva etapa.

Hablar de observación participante sería dar cuenta de nuestra disposición general, pero no de las actividades concretas que llevamos a cabo en el campo; hablar de entrevistas en profundidad supondría evocar una situación que pocas veces se nos planteó con tanta nitidez y delimitación; y referirnos a información nos retrotraería a una objetivación con la que nos vinculamos que no estaríamos dispuestas a admitir porque no da cuenta de qué sentimos y cómo vivimos nuestro trabajo de campo con otras personas (Guber, 2014, p.15).

La escucha y la observación se convirtieron en herramientas fundamentales de acercamiento y sistematización. Este adaptarme a los tiempos de la organización se tradujo en escucha y observación sin registro de audio o escrito en el momento. La narración de experiencias de este tipo produce un quiebre en el lenguaje que imposibilita -efectivamente- el relato. Todo es silencio, hueco, vacío. Situar las preguntas de un investigador en estos lugares significa imponer ritmos y formatos narrativos para el contar, por ende, rememorar, volver a vivir, supone intervenir en tiempos subjetivos del recuerdo horroroso y sufrimiento de la experiencia; busca gestionar lo indecible, rellenar los huecos narrativos, encontrar aquello inaprensible. No podía dejar (aún no) de ver la presencia de un investigador en estas tramas como algo intrusivo, y por ello buscamos la forma de investigar con una metodología que contemplara estos cuidados.

Cuando se habla sobre violaciones, se le da una gran importancia a los silencios. Qué hacer con estos silencios -cómo escucharlos, cómo interpretarlos, cómo determinar cuando son opresivos y cuando pueden constituir una forma de agenciaes un tema de gran preocupación y debate (Theidon, 2006, p. 71).

Aun así, esta propuesta nos abre otros interrogantes: ¿en qué medida las organizaciones que son facilitadoras de la enunciación de los relatos de estas víctimas permiten efectivamente la emisión de esta voz?; ¿qué géneros habilitan para moldear el sufrimiento y el dolor?; ¿puede narrarse el horror con estos géneros disponibles para el contar?; ¿¿en qué medida son compatibles estos géneros disponibles con los que necesita la justicia para proceder a favor de las víctimas? Decidí (no antes de presenciar estas lógicas) que la mejor manera de estudiar los relatos era a partir de la escucha y la observación. Nada más. Y nada menos.

Theidon, quien participó en las Comisiones por la Verdad y la Reconciliación en Perú (CVRP) en el $2003^{1}$ , trabaja con relatos enunciados en grupos focales, ya que también considera que el contexto de enunciación y recepción de estos testimonios es determinante de las formas discursivas: "me interesaban este tipo de recuerdos relatados en estos grupos focales porque el contexto en el cual los testimonios son dados y recibidos es central con relación a las formas que esos testimonios adquieren” (Theidon, 2006, p. 82). Esto y la constante pregunta por la ética en campo: ¿tenemos derecho a preguntar sobre experiencias traumáticas?; ¿hay límites en investigación social?; ¿cuál es la metodología "menos" violenta, invasiva, entrometida para el estudio de experiencias traumáticas? "No puedo separar los métodos de la ética: en este caso, ambos son repugnantes. Hay preguntas que no tenemos derecho a preguntar, y silencios que deben ser respetados" (Theidon, 2006, p. 87).

El silencio fue una constante en estas narrativas, su decodificación fue un aprendizaje diario. "Cuando termine de contar, ¿̇me voy a seguir acordando de lo que pasó?”, preguntó una joven a la terapeuta en una de las sesiones. $Y$ es que el silencio no entraba en los géneros disponibles para el contar. Tuvimos que aprender a convivir con los silencios, percatadas de que eran una constante en trabajos de este tipo: "Cuando me olvido 
me siento bien. Recordar (lo que pasó) incluso ahora, me vuelve loca. Se me hace muy difícil poder soportarlo. Pero cuando me olvido me siento más o menos. Es tan duro responder a sus preguntas, tan difícil volver atrás y recordarlo todo" (Theidon, 2006, p. 82).

Romero plantea que "la ruptura de las condiciones de posibilidad de la comprensión de hechos de degradación y muerte, la necesidad de hablar, la urgencia de ser escuchado, la emergencia del silencio para preservar la intimidad o el anonimato, el hueco, el vacío, el mismo dolor" (Romero, 2008, p. 21) son sentires encontrados con los de una investigación que busca escribirse. Las urgencias del investigador por acceder a esas palabras y la impaciencia de esa manera de hacer investigación (la mayoría de las veces) son incompatibles con los tiempos de las personas que intervienen en la misma. Entonces, el desafío del reflexionar metodológico radica precisamente allí, en la búsqueda de herramientas y técnicas que respeten, en lo posible, los tiempos de los/as otros/as, sus espacios y seguridades. Implica reconocer y detectar qué nos falta antes de ingresar a campo, ¿es sólo una preparación teórica- metodológica?; ¿son sólo los años de experiencia en investigación? ¿O es quizás una preparación que tiene que ver con sostener el cuerpo y prestar atención a las sensibilidades que se juegan en ese momento?

Si investigadores en comunicación sostienen que habilitar espacios de charla (entrevistas) es habilitar la posibilidad de poner en palabras el horror y terminar de vivir la experiencia: ¿cómo entonces se intenta "reparar" lo no-dicho?

\section{(IN)CONCLUSIONES}

¿Es legitimo investigar narrativas que tienen que ver con el dolor del otro?; ¿de qué manera nos interpela ese dolor?; ¿qué lugar tenemos como comunidad académica en estas nuevas cartografias de lucha?; ¿qué podemos ofrecer al esclarecimiento de nuestros interrogantes y a sus interrogantes?; trabajos como éstos, ¿contribuyen/ mejoran/intervienen de manera favorable a las victimas?; ¿cómo poner este bagaje y accionar reflexivo al servicio de las organizaciones que trabajan en la búsqueda de justicia y reparación?. En definitiva, ¿podría existir un uso instrumental de las conclusiones de trabajos como éstos para situaciones concretas?

Iván Bondar -doctor, investigador del CONICET especialista en estudios que tienen que ver con la antroposemiótica de la muerte y el morir-, en ocasión de un panel reflexivo sobre el femicidio de Lucia Maidana $^{2}$ al cual fui invitada a exponer, responde la pregunta que abre este último apartado: “¿Sabés por qué es legítimo trabajar con el dolor ajeno?”. Me pregunta en tono reflexivo, cauteloso: "Porque como investigadores habilitamos espacios para que se descarguen y puedan hablar de quien perdieron. Durante las entrevistas que duran horas pueden contar lo que atravesaron y desahogarse". A esta intervención, la Mgter. Elena Maidana ${ }^{3}$ responde:

Eso desde el punto de vista de un metodólogo, nosotras en Comunicación preferimos hablar de la habilitación de espacios de charla que permitan terminar de cerrar o terminar de vivir la experiencia... se traduce en escucha en nosotras pero habilita la palabra al otro.

Este intercambio en términos metodológicos que se dio en este panel fue, de alguna manera, la puerta para empezar a responder (o repreguntar) sobre estas cuestiones: ¿Puede la comunicación, como disciplina, contribuir metodológicamente a lograr una ciencia más sensible, comprometida, cercana a sus pueblos?

Como investigadoras, la única manera que encontramos de lidiar con las condiciones de del campo fue la de proponer una metodología que dialogara de cerca con las formas y tiempos de la organización, en este caso la ONG. Los registros se limitaron a ser lo menos intrusivos que fuera posible, siempre con toma de notas posterior al encuentro. La escucha y observación fueron las principales estrategias para abordar esta red de relaciones que se mostraba por momentos trunca e inaccesible.

La metodología debería ser pensada en función del campo o de la posibilidad del campo que se nos presentara. La "vigilancia epistemológica" sobre la que hablaba Guber también tiene que ver con pensar y 
reflexionar a partir de otras organizaciones y organismos que abordan de una u otra manera la problemática (que deviene en problema de investigación). Esta propuesta contribuyó a pensar el trabajo articulado en la tesis, y cómo podemos proponer modos de encarar el campo de manera menos intrusiva, a través y a partir de las lógicas organizacionales de cada lugar. Un abordaje ético supone esto: la reflexión sobre las formas de acceder y tratar toda información que supondrá un dato, y también la asimilación de la idea de que no todo puede ser registrado en campo, y de que no toda información es sensible de ser convertida en dato para un trabajo científico.

\section{ReFERENCIAS}

Achío Tacsan, M. (2003). Los comités de ética y la investigación en Ciencias Sociales. Revista En Ciencias Sociales, I, 85-95.

Bajtin, M., y Voloshinov, V. (1998). ¿Qué es el lenguaje? La construcción de la enunciación. Ensayo sobre Freud. Buenos Aires: Editorial Almagesto.

Bialakowsky, A.; Franco, D.; Patrouilleau, M.; Bardi, N., y Lusnich, C. (2009). Homo faber: esculpiendo hombres. La coproducción investigativa en la interioridad de una práctica. Convergencia, 16(51), septiembre-diciembre, 183-212.

De Sousa Santos, B. (2009). Pensar el estado y la sociedad: desafios actuales. Buenos Aires: Waldhuter Editores.

Figari, C., y Haber, A. (2010). Conocimiento situado y técnicas amorosas de la ciencia. Tópicos de epistemología critica. Recuperado https://epistemologiascriticas.files.wordpress.com/2011/05/figari_conoc-situado.pdf

Guber, R. (comp.) (2014). Prácticas Etnográficas. Ejercicios de reflexividad de antropólogas de campo. Buenos Aires: Miño y Dávila Editores.

Guber, R. (2013). La articulación etnográfica. Descubrimiento y trabajo de campo en la investigación de Esther Hermitte. Buenos Aires: Editorial Biblos.

Guber, R. (2005). El salvaje metropolitano. Reconstrucción del conocimiento social en el trabajo de campo. Buenos Aires: Editorial Paidós. Estudios de Comunicación.

Haber, A. (2011). Nometodología payanesa. Notas de metodología indisciplinada. Revista de Antropología, 23, 9-49. Romero Aranguren, J. P. (2008). El investigador ante lo indecible y lo inenarrable. Revista Nómadas, 29, 20-33.

Vasilachis De Gialdino, I. (2006). La investigación cualitativa. En Estrategias de Investigación cualitativa, 23-64. Barcelona: Gedisa Editorial.

Theidon, K. (2006). Género en transición: sentido común, mujeres y guerra. Cuadernos de Antropología Social, 24, 69-92.

\section{Notas}

1. La Comisión tenía por objetivo examinar las causas y consecuencias del conflicto armado interno que tuvo lugar entre los 80 y 90 en Perú. De esta manera, Perú se unió a la creciente lista de países que habían implementado comisiones de verdad como medio de transición de un período de conflicto armado y gobierno autoritario hacia la fundación de una democracia procesual.

2. Lucia Maidana era estudiante de la carrera de Comunicación Social de mi universidad. Compañera de la carrera, unos años más chica que yo, fue golpeada, violada y prendida fuego en su vivienda del barrio El Palomar de la ciudad de Posadas, el 6 de abril del 2013. El crimen involucra a los mismos alumnos de la carrera de Comunicación, "compañeros" que recorrieron los mismos pasillos que Lucía y hoy recorren los mismos pasillos que nosotras. Hasta el momento en que estas líneas fueron escritas no había detenidos ni avance alguno en la causa.

3. Exdocente de la carrera de Licenciatura y Tecnicatura en Comunicación Social de la Facultad de Humanidades y Ciencias Sociales de la Universidad Nacional de Misiones, fue una de las principales impulsoras de la orientación en investigación en la cual me formé en grado. Investigadora de la FHyCS en temas referidos a ciudad y comunicación. 